\title{
CONSULTATION SUR LE CONTROLE BIOLOGIQUE DES MOLLUSQUES VECTEURS
}

Une consultation informelle, la première de ce genre, sur la Recherche pour un Contrôle Biologique des Mollusques hôtes intermédiaires, s'est tenue à Genève (Suisse) du 25 au 27 janvier 1984, sous l'égide du Programme Spécial de Recherche et de Formation sur les Maladies Tropicales (TDR) de l'UNDF/Banque Mondiale/OMS, et, en particulier, des groupes de travail sur le Contrôle Biologique des Vecteurs (SWG/BCV) et sur les Schistosomoses (SWG/SCH).

Les objectifs de cette consultation étaient les suivants :

- établir un bilan de l'état de la recherche et de la mise en œuvre sur le terrain du contrôle biologique des Mollusques hôtes intermédiaires en général et notamment du rôle de la compétition entre Mollusques;

- développer un schéma permettant de tester sur le terrain les Mollusques compétiteurs comme agents de contrôle, schéma tenant compte des voies souhaitables de recherche, des règles de sécurité et des besoins en formation ;

- mettre en évidence les principaux facteurs, aussi bien théoriques que pratiques, qui influencent le rapport coût-efficacité du contrôle biologique dans les programmes de lutte contre les Mollusques ;

- mettre au point des recommandations pour le suivi des opérations.

Pour le besoin de la Consultation, le terme " contrôle biologique " a été défini comme "l'usage d'organismes vivants antagonistes des Mollusques hôtes afin d'interrompre le cycle de transmission à haut niveau des Schistosomoses humaines ».

Les participants ont conclu que le contrôle biologique des Mollusques hôtes pouvait, dans certains types de sites de transmission, améliorer de façon significative le rapport coût-efficacité du contrôle des Schistosomoses. Recherches de terrain plus rigoureuses et formation adéquate permettraient d'exploiter cette approche. Les participants ont proposé une base théorique pour développer des stratégies de contrôle biologique; ils ont pris en considération l'information actuellement disponible sur les quatre groupes d'antagonistes des Mollusques hôtes, à savoir les pathogènes, les parasites, les prédateurs et les compétiteurs. Des lignes directrices ont été tracées pour des recherches sur l'efficacité, l'inocuité et la mise en œuvre opérationnelle des agents de contrôle biologique.

Des priorités d'ordre général ont été définies et des recommandations spécifiques à chacun des quatre groupes ont été formulées. Les aspects relatifs à la sécurité (qui peut être un obstacle majeur à l'utilisation de certains agents de contrôle) et au coût ont été traités.

Le rapport de cette réunion a pour objet d'aider les scientifiques à planifier leur recherche dans le domaine du contrôle biologique des Mollusques vecteurs. Il pourra être un guide pour tout projet d'importation et de lâcher d'organismes allochtones pour le contrôle des Mollusques.

Le rapport est disponible sur demande auprès du directeur du Programme TDR, OMS, 1211 Genève 27 (Suisse). 